Eur Radiol. Author manuscript; available in PMC 2016 April 19.

Published in final edited form as:

Eur Radiol. 2015 November ; 25(11): 3282-3294. doi:10.1007/s00330-015-3731-2.

\title{
Cost-Utility Analysis of Nonalcoholic Steatohepatitis Screening
}

Eric Zhang, MD ${ }^{1,5}$, Claire Wartelle-Bladou, MD $^{2}$, Luigi Lepanto, MD, MSc ${ }^{1,3}$, Jean Lachaine, PhD $^{4}$, Guy Cloutier, PhD, PEng ${ }^{1,5}$, and An Tang, MD, MSc ${ }^{1,5}$

${ }^{1}$ Department of Radiology, Radio-Oncology and Nuclear Medicine, University of Montreal, SaintLuc Hospital, Montreal, Quebec, Canada

${ }^{2}$ Department of Gastroenterology and Hepatology, University of Montreal, Saint-Luc Hospital, Montreal, Quebec, Canada

${ }^{3}$ Health Technology Assessment Unit, University of Montreal, Saint-Luc Hospital, Montreal, Quebec, Canada

${ }^{4}$ Faculty of Pharmacy and Pharmacoeconomics, University of Montreal, Montreal, Quebec, Canada

${ }^{5}$ Laboratory of Biorheology and Medical Ultrasonics, University of Montreal Hospital Research Center (CRCHUM), Montreal, Quebec, Canada

\section{Abstract}

OBJECTIVES-Nonalcoholic fatty liver disease (NAFLD) is the most common liver disease in Western countries. No studies have examined the cost-effectiveness of screening for nonalcoholic steatohepatitis (NASH), its advanced form.

METHODS-We performed a cost-utility analysis of annual non-invasive screening strategies using third-party payer perspective in a general population and compared it to screening in a highrisk obese or diabetic population. Screening algorithms involved well-studied techniques including NAFLD fibrosis score, transient elastography (TE), and acoustic radiation force impulse (ARFI) imaging for detecting advanced fibrosis ( $\geq \mathrm{F} 3$ ); and plasma cytokeratin (CK)-18 for NASH detection. Liver biopsy and magnetic resonance elastography (MRE) were compared as confirmation methods. Canadian dollar costs were adjusted for inflation and discounted at $5 \%$.

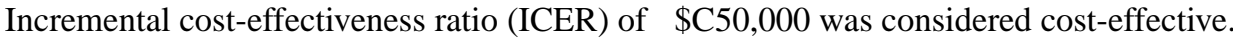

RESULTS-Compared with no screening, screening with NAFLD fibrosis score/TE/CK-18 algorithm with MRE as confirmation for advanced fibrosis had an ICER of \$C26,143 per quality-

Corresponding author information: An Tang, MD, MSc, Department of Radiology, Faculty of Medicine, University of Montreal, Saint-Luc Hospital, 1058 rue Saint-Denis, Montréal, Québec Canada, H2X 3J4, Telephone: (514) 890-8000 \#35607, Fax: (514)

412-7359, an.tang@umontreal.ca.

Author contributions/emails:

Eric Zhang: Analysis and interpretation of data, drafting of the manuscript, critical revision of the manuscript, approved final submission. eric.zhang@mail.mcgill.ca

Claire Wartelle-Bladou: Critical revision of the manuscript, approved final submission. clairewartelle@gmail.com

Luigi Lepanto: Study concept and design, critical revision of the manuscript, approved final submission. luigi.lepanto@umontreal.ca Jean Lachaine: Critical revision of the manuscript, approved final submission. jean.lachaine@ umontreal.ca

Guy Cloutier: Critical revision of the manuscript, approved final submission. guy.cloutier@umontreal.ca

An Tang: Study concept and design, analysis and interpretation of data, drafting of the manuscript, critical revision of the manuscript, obtained funding, study supervision, approved final submission. an.tang@umontreal.ca 
adjusted life year (QALY) gained. Screening in high-risk obese or diabetic populations was more cost-effective, with an ICER of \$C9,051 and \$C7,991 per QALY gained respectively. Liver biopsy confirmation was not found to be cost-effective.

CONCLUSIONS-Our model suggests that annual NASH screening in high-risk obese or diabetic populations can be cost-effective.

\section{Keywords}

Cost-effectiveness; Nonalcoholic fatty liver disease (NAFLD); Fibrosis; Elastography; Screening

\section{INTRODUCTION}

Over the last decade, nonalcoholic fatty liver disease (NAFLD) has been recognized as the most prevalent liver disease in Western countries, due in large part to the high rates of obesity and type 2 diabetes [1]. It affects an estimated $20-30 \%$ of the general adult population, and as much as $90 \%$ of diabetic or obese patients [2-4]. The more advanced form, nonalcoholic steatohepatitis (NASH) may evolve to fibrosis, cirrhosis, liver failure, and hepatocellular carcinoma (HCC) [5-9].

Although healthcare costs related to NASH have not been well studied, they are estimated to be significant due to potential progression to liver failure and HCC. NASH-related liver failure is predicted to become the main cause of liver transplantation within the next decade [10]. Current practice guidelines do not advocate screening of NAFLD or NASH at this time, in part due to a lack of knowledge regarding optimal non-invasive diagnostic strategies, long-term benefits, and the cost-effectiveness of screening [11; 12]. Although liver biopsy is the current reference standard for diagnosis of steatohepatitis and advanced fibrosis in patients with NAFLD [12], its invasiveness makes it an unlikely modality for large-scale screening [13]. To address this issue, non-invasive blood tests and elastography methods have been introduced for the detection of NASH or advanced fibrosis ( $\geq F 3)$ [14-16]. Screening for this highly prevalent disease may be worthwhile [17], but as of yet, the value of screening strategies for NASH has not been studied.

Weight loss is currently the recommended standard of care for NASH [12; 18]. In more advanced disease, vitamin $\mathrm{E}$ has been recommended as pharmacotherapy in non-diabetic patients with histologically-proven NASH $[11 ; 12]$. Pioglitazone is another suggested pharmacotherapy based upon latest randomized-control trials and meta-analyses [19; 20]. A recent cost-utility study established the cost-effectiveness of pharmacological therapy for delaying the progression of NASH fibrosis using pioglitazone and vitamin E [21].

To our knowledge, there is currently no cost-utility study for NASH screening in the Western population. In this era of cost-containment, cost-effectiveness and opportunity cost of screening for NASH must be investigated. Thus, the primary aim of our study was to estimate the cost-effectiveness in a general population of different screening strategies for NASH or advanced fibrosis ( $\geq \mathrm{F} 3$ ) detection, while incorporating currently recommended treatment practices. In order to determine the optimal population to target for screening, our 
secondary aim was to estimate the cost-effectiveness of these screening strategies in highrisk obese or type 2 diabetes populations.

\section{MATERIALS AND METHODS}

\section{Markov Model and Assumptions}

From a health-care system perspective, a decisional Markov model [22] was developed (TreeAge Software, Williamstown, MA) to estimate the expected lifetime costs and QALYs associated with screening strategies for NASH. This model was constructed to mirror the natural history of NAFLD disease progression through the histopathological continuum of simple steatosis, NASH, fibrosis stages, and cirrhosis [5]. Patients with cirrhosis may progress onto liver failure and also have increased probability of developing HCC (Figure 1) [23].

To address our research aims, we ran the simulation for a general population and for highrisk populations, either with obesity or type 2 diabetes. Patients began screening at the age of 30. At the beginning of the simulation, the population was divided among these mutually exclusive health states according to mean prevalence rates reported in developed countries for a general population, an obese population, and a type 2 diabetes population, respectively. The model assumed an annual cycle length. In each cycle, simulated populations could remain in their health states or progress according to transition probabilities derived from literature. Screening and treatment strategies were superimposed onto this life cycle model of NAFLD. Before screening began, the costs of specialist consultation and laboratory tests to rule out alternate causes of chronic liver injury were taken into account. For the purpose of developing this model, histological improvement was assumed a good correlate for clinical outcomes. Both all-cause and liver-related mortalities were taken into account at each stage of disease. The simulation ended once every member of the population died. A lifetime horizon was chosen for this model to better reflect NAFLD disease progression [24], as well as to better represent the magnitude of costs and utilities associated with the disease. Peer-reviewed guidelines for economic evaluations were followed in the creation of this model [22; 25].

\section{Competing Screening Strategies}

The competing screening strategies incorporated independently and widely studied noninvasive tests. Plasma cytokeratin-18 (CK-18) was assessed for the noninvasive detection of NASH. NAFLD fibrosis score, ultrasound transient elastography (TE), and ultrasound acoustic radiation force impulse (ARFI) imaging were assessed for the detection of advanced fibrosis ( $\geq F 3)[14 ; 16 ; 26]$. We compared a sequential algorithm [14] that incorporates the NAFLD fibrosis score, transient elastography, and CK-18 with biopsy confirmation to no screening in our Markov model. The underlying assumption was that a strategy combining noninvasive methods for NASH and fibrosis detection would decrease the number of unnecessary liver biopsies [14; 16]. Given the similar sensitivity of ultrasound-based elastography for the detection of advanced fibrosis, we compared a variant of this sequential algorithm by substituting TE with ARFI [27]. In addition, considering the high diagnostic accuracy of magnetic resonance elastography (MRE) for fibrosis staging 
[26], we also compared MRE against liver biopsy for the confirmation of advanced fibrosis. The mortality risk associated with liver biopsy as well as the costs associated with severe bleeding complications were implemented in the model [28]. Figure 2 illustrates the various screening strategies compared in our study.

\section{Treatment Arms}

Three treatment branches were implemented in the model. In accordance with international guidelines, NASH patients with no or mild fibrosis $(\mathrm{F} \leq 1)$ were treated with lifestyle intervention and weight loss, whereas patients with advanced fibrosis $(F \geq 3)$ were treated pharmacologically [29]. Lifestyle intervention aimed to achieve an overall weight reduction of 7-10\% by combining regimented exercise, diet, and behavior adjustments. The treatment effect on NASH progression was calculated from a randomized controlled trial looking at the histological improvements of a lifestyle intervention program versus standard of care [18]. The pharmacotherapies considered in our model included vitamin $\mathrm{E}$ and pioglitazone $[19 ; 30]$. Treatment effects on fibrosis progression were estimated by applying the relative risk for histological improvement used in a previous cost-utility analysis [21]. Pharmacotherapies were stopped in the event of liver decompensation development, in accordance with assumptions made previously [21].

\section{Model Parameter Estimates}

Prevalence, annual transition probabilities, and mortality risk for the Markov model were derived from a systematic literature review (Table 1). Annual transition probabilities were calculated based on the approach outlined by Miller and Homan for converting rates over time [31]. Screening test sensitivities were obtained from meta-analyses. Liver biopsy, as the accepted reference standard, was assumed to have $100 \%$ accuracy.

\section{Costs}

Annual healthcare costs were derived from the Canadian Provincial Billing Guides [32]. Relevant costs include primary care follow-up, specialist consultation, and blood work panels to rule out alternative diagnoses of chronic liver disease. Screening tests were microcosted from the Canadian Provincial Billing Guides, the Canadian Agency of Drugs and Technologies in Health, and related literature on micro-costing of elastography methods in Canada [33-35]. Cost of cytokeratin-18 M30-apoptosense ELISA kit (PEVIVA, Bromma, Sweden) were obtained from the company website [36]. Annual patient care costs for liver decompensation were taken from the Canadian Institute of Health Information [37]. The costs of HCC management and liver transplantation were derived from published literature specific to the Canadian healthcare system [38; 39]. All costs incorporated into the model are in 2013 Canadian dollars (\$CAD) (Table 2 and Supplementary Table 1). Costs were adjusted for inflation to 2013 when needed using the national inflation index [40].

\section{Health-Related Quality of Life}

For health-related quality of life data, we used the largest study performed in patients with NAFLD to date [41]. This study provided quality of life data on patients with NAFLD and NASH in the form of a SF-36 survey. The data from these surveys were then converted to 
utility estimates using the method described by Nichol et al. [42]. Further health-related quality of life information on NASH-associated fibrosis, cirrhosis and hepatic decompensation were nonexistent. Therefore, we used utilities from health-related quality of life studies on other causes of chronic liver disease [43-46]. Given the benign nature of the disease, simple steatosis was assumed an utility estimate of 1 . Utility values for each health state are reported in Table 3.

\section{Outcomes}

Outcomes were measured in terms of costs (\$CAD) and in terms of quality-adjusted life years gained (QALYs). The incremental cost-effectiveness ratio (ICER) of each strategy was calculated as the incremental difference in cost divided by the incremental difference in quality-adjusted life years of two consecutive strategies. In the Canadian heath care setting, ICERs of less than $\$ C 50,000$ per QALY gained is usually considered cost-effective. The discount rate was set at 5\% in accordance with Canadian guidelines [25]. A strategy is dominating when it results in lower cost and higher QALYs in comparison to another and dominated when it results with higher cost and less QALYs in comparison to another.

\section{Sensitivity Analyses}

The robustness of our results was assessed in terms of one-way sensitivity analyses, in which all model parameters were varied across a range taken from published data or at 95\% confidence intervals. For the transition probability from simple steatosis to NASH, which was not readily available due to a paucity of data, we took a large range of plausible values [24]. Two-way sensitivity analyses were performed on select pairs of parameters that were influential in one-way sensitivity analyses. There were not enough published data to build probability distributions for undergoing a probabilistic sensitivity analysis.

\section{RESULTS}

Table 4 illustrates the results for the top three dominating screening strategies for the base case analysis for each population studied. In the general population, no surveillance as a baseline strategy costs $\$ C 6,561$ per person with a total utility value of 42.04 QALYs gained over the lifetime of the patient. NAFLD fibrosis score/TE/CK-18 sequential strategy with MRE confirmation for advanced fibrosis and vitamin E treatment cost $\$ C 3,136$ more per person but also delivered incremental utility increase of 0.12 QALYs. This strategy was found to be cost-effective with an ICER of \$C26,143/QALY gained according to a threshold of $\$ C 50,000 / \mathrm{Q} A L Y$ gained. The same strategy with pioglitazone treatment was found to have an ICER of \$C199,870/QALY gained.

\section{Cost-utility in High-risk Populations}

In an obese population, the NAFLD fibrosis score/TE/CK-18 sequential strategy with MRE confirmation for advanced fibrosis and vitamin E treatment resulted in an ICER of \$C9,051/ QALY gained compared to no surveillance. In a type 2 diabetic population, the same screening strategy resulted in an ICER of \$C7,991/QALY gained compared to no surveillance. The remaining screening strategies not seen in Table 4 were dominated and therefore not found to be cost-effective. 


\section{Sensitivity Analyses}

One-way sensitivity analysis results for the NAFLD fibrosis score/TE/CK-18 sequential algorithm with MRE confirmation and vitamin E treatment are summarized in Figure 3. In this analysis, all parameters used during the simulations were varied through the range of values found in literature or by applying 95\% confidence intervals to test the robustness of our results given the potential uncertainty of parameter values. The ICER for the base case scenario is delineated by the vertical line. The ICERs within the variable range tested move from the blue (lower range) to the red side (upper range).

Three variables were found to have the greatest effect on the ICER: the test cost for TE, the starting age for screening, and the annual transition probability of steatosis to NASH. If the cost of an individual TE test was assumed to be \$C50, then the ICER was \$C20,521/QALY gained. At an upper limit assumption of $\$ C 250$ per test, then the ICER increased to $\$ C 43,040 / \mathrm{QALY}$ gained. If the starting age for screening began at 18 years of age, the ICER was found to be as low as $\$ C 17,535 / \mathrm{QALY}$ gained. However, if screening began at 43 years old or later, the ICER surpassed the \$C50,000/QALY gained threshold. If the annual probability of developing steatohepatitis was $8.8 \%$, then the ICER would be \$C11,164/ QALY gained; however, if the annual incidence of steatohepatitis were as low as $0.03 \%$, then the ICER would increase to more than \$C42,787/QALY gained.

In accordance with Canadian health technology assessment guidelines, the model was assessed using $0 \%$ as well as 3\% discount rates for comparison purposes with other jurisdictions [25]. In general, lowering the discount rate resulted in more cost-effective strategies. At $0 \%$ discount, all screening strategies became more cost-effective. The NAFLD fibrosis score/TE/CK-18 sequential algorithm with MRE confirmation resulted in an ICER of \$C15,493/QALY gained.

Two-way sensitivity analyses were conducted to examine the effect on ICER of varying pairs of influential variables simultaneously. This can help distinguish particular thresholds whereby one strategy becomes more cost-effective than another assuming a $\$ C 50,000 /$ QALY gained threshold. Two-way sensitivity analyses found that ARFI and TE were interchangeable in the sequential algorithm.

\section{DISCUSSION}

We performed a cost-utility analysis to address the current knowledge gap regarding the cost-effectiveness of screening strategies for steatohepatitis and NASH-fibrosis, two advanced forms of NAFLD that may progress to end-stage liver disease. By combining the most widely studied non-invasive tests, we categorized patients according to their probability of having advanced disease and thus limit the total number of liver biopsies [14; 16]. To further decrease the invasiveness of a screening strategy for NASH, we examined the potential of MRE as an alternate reference standard to liver biopsy for liver fibrosis diagnosis, based on promising meta-analysis results [26]. Finally, we compared these screening algorithms in both general and high-risk populations to determine the most costeffective population to screen. 
Our model suggests that, in a general population, a sequential algorithm that includes the NAFLD fibrosis score/TE/CK-18, with MRE confirmation for advanced fibrosis, and vitamin $\mathrm{E}$ as treatment, can be a cost-effective surveillance strategy with an ICER of \$C26,143/QALY gained. In comparison, the same sequential algorithm with pioglitazone treatment had a higher ICER of \$C199,870/QALY gained. The results indicate that the combination of non-invasive tests for detection of advanced fibrosis and NASH, with lifestyle changes and vitamin $\mathrm{E}$ as treatment, provides incremental gains of QALYs over no surveillance. By detecting earlier stages of the NAFLD and by implementing treatment according to current guidelines, this surveillance strategy demonstrates the potential to limit the transition of patients towards liver cirrhosis and end-stage liver disease, and its associated quality-of-life and economic costs.

Our model suggests that MRE is more cost-effective than liver biopsy as a confirmation method in a screening program for advanced fibrosis $(\geq F 3)$. Strategies with liver biopsy as confirmation for advanced NASH-fibrosis were more costly for less QALYs gained. This result reflects both the potential of MRE as an alternative reference standard, as well as the mortality and morbidity associated with liver biopsy. In recent years, MRE has emerged as a highly accurate modality for the staging of liver fibrosis, with histopathology as the reference standard [26]. From the point of view of a screening program, confirmation with MRE would likely be better accepted by the general population, given that it is non-invasive.

The key drivers of cost-effectiveness were the cost of TE, the starting age of surveillance, and the annual transitional probability from simple steatosis to NASH. In the base case scenario, the underlying assumption was that screening would begin at 30 years of age. Given that NASH and its complications are becoming an increasing problem among younger people [12], earlier screening could be a possibility. Ultrasound-based elastography methods, namely TE or ARFI, have similar sensitivities for detection of advanced fibrosis $[27 ; 47 ; 48]$ and may be used interchangeably for fibrosis staging in the clinical workflow [16]. However, in our model, a sequential algorithm in which TE was substituted with ARFI was found to be dominated by the leading screening strategy with TE. This difference in cost/QALY gained may be explained by the higher cost of ARFI over TE in our microcosting scenario. Since itemized costs for these elastography tests have yet to be established in the Canadian healthcare billing guides, the micro-costing relied on a series of assumptions. To address the inherent uncertainties surrounding our assumptions on costs for these exams, we performed a two-way sensitivity analysis, which suggested that ARFI and TE were close to equivalent in the sequential screening algorithm along the range of costs from $\$$ C50 to $\$ C 250$.

While TE and ARFI appear to be similar in their sensitivity and cost-effectiveness, they do have unique advantages and disadvantages. For example, transient elastography devices (Fibroscan) may also detect for liver steatosis using the Controlled Attenuation Parameter (CAP) [49; 50]. CAP represents a promising adjunct for the quantification of liver steatosis alongside fibrosis at the same time. However, TE suffers in the detection of fibrosis in obese patients, with failure rates and unreliable results that are higher than in non-obese patients. This disadvantage can be partially circumvented, however, by using the XL probe, although at an additional cost $[51 ; 52]$. On the other hand, ARFI technique is coupled with imaging 
and permits detection of liver fibrosis even in patients with a large body habitus. Some studies have thus far demonstrated that ARFI was feasible in obese patients and provide diagnostic accuracy similar to that of TE with the XL probe for the staging of liver fibrosis [53-55].

Our secondary aim was to examine the cost-effectiveness of these same screening strategies in high-risk obese and type 2 diabetes populations. We found that the most cost-effective screening strategy in a general population (\$C26,143/QALY gained) was significantly more cost-effective in high-risk populations ( $\$ C 9,051 / \mathrm{QALY}$ gained in an obese population and $\$ C 7,991 /$ QALY gained in a type 2 diabetes population).

One of the principles of preventing over-diagnosis is to better differentiate between benign disease and progressive disease that will cause more harm [56]. Thus, in our model, we did not screen for simple steatosis because, without inflammation, it is considered a benign, non-progressive disease in the majority of patients and not likely to develop into advanced fibrosis during their lifetimes [57]. We have not included steatosis screening in our Markov model because only a small subset of patients with steatosis ever progress to NASH and cirrhosis $[24 ; 58]$. Also, patients with simple steatosis have a survival similar to that of the general population, whereas patients with NASH have a higher overall mortality [14; 59]. While simple steatosis is a hallmark feature of early NAFLD, it may be replaced by fibrosis in advanced disease [60; 61]. Finally, we based our cost-utility analysis on prior screening algorithms published in literature, which did not emphasize liver fat screening for these reasons [14].

Instead, we focused on the non-invasive detection of steatohepatitis and fibrosis, both of which are progressive stages of NAFLD and can lead to major complications if not found and treated. In our model, the non-invasive detection of NASH without advanced fibrosis depended on CK-18 fragments, which has a fair accuracy for NASH screening [62], with confirmation by liver biopsy. The current challenge with CK-18 includes its limited availability and as such it has not been introduced in clinical practice in Canada.

Alternatively, MRE has been proposed for detection of NASH [63]. However, this will require independent validation in the future before we can consider an entirely non-invasive screening algorithm.

There are limitations to our study. The relevance of screening relies on the assumption that effective long-term therapy for NASH exists. It is conceivable that the histological improvements observed in short-term randomized controlled trials on lifestyle modification [18], pioglitazone, and vitamin E [19; 20] may not be sustainable after discontinuation of therapy and over the lifetime horizon. Thus, longer-term studies on NASH and antifibrotic treatment are required. Nonetheless, current guidelines advocate pharmacotherapy (vitamin $\mathrm{E}$ and glitazones) with caution in specific patients with elevated risk of progression to cirrhosis who have failed lifestyle intervention [11].

Further, we did not model the potential side effects of pharmacotherapy. Glitazones have been implicated in long-term safety concerns regarding cardiovascular disease, bladder cancer, and bone loss whereas vitamin $\mathrm{E}$ has been associated with a possible increase in all- 
cause mortality and risk of prostate cancer. However, given that there has been much controversy and conflicting results in the literature [64-68], and that it was not possible to model all complications for the purposes of an economic model, we decided not to implement them.

Our study has the following strengths. The algorithms studied in our model were derived from meta-analyses and compatible with current guidelines. The model parameters were based on a systematic literature review to identify prevalence, transition probabilities, costs, and utilities. These parameters represent a comprehensive simulation of NAFLD continuum. Where possible, we used utility estimates for steatohepatitis derived from a population with NASH [41].

In summary, our cost-utility model suggests that NASH screening is cost-effective with noninvasive screening methods for steatohepatitis and advanced fibrosis. Furthermore, screening in high-risk populations of obese or type 2 diabetes patients is more cost-effective than in a general Western population. Before decision-makers decide to implement a screening program, further studies should better establish the quality of life in NASH and the longterm effectiveness and safety of therapy.

\title{
Supplementary Material
}

Refer to Web version on PubMed Central for supplementary material.

\section{Acknowledgments}

The abstract of this study has been previously presented at the European Congress of Radiology (ECR) 2014 and the International Society for Pharmacoeconomics and Outcomes Research (ISPOR) 17th Annual European Congress in Amsterdam, Netherlands on 10 November 2014.

The scientific guarantor of this publication is Dr. An Tang. The authors of this manuscript declare no relationships with any companies, whose products or services may be related to the subject matter of the article.

\begin{abstract}
This study has received funding by (1) Canadian Institutes of Health Research - Institute of Nutrition, Metabolism and Diabetes (CIHRIMND) Operating Grant (\#301520); (2) Fonds de recherche du Québec en Santé (Junior 1 Career Award FRQS-ARQ \#26993 to An Tang, MD); and (3) Recruitment fund for new scientist at the CRCHUM to An Tang. Funding agencies did not have any role in the design and conduct of the study, collection, management, analysis or interpretation of the data, preparation, review, or approval of the manuscript.
\end{abstract}

No complex statistical methods were necessary for this paper.

Institutional Review Board approval was not required because the research project did not involve human or animal subjects. None of the study subjects or cohorts have been previously reported.

Methodology: Costeffectiveness study performed at one institution

\section{ABBREVIATIONS AND ACRONYMS}
ARFI
acoustic radiation force impulse
CK-18
cytokeratin-18
HCC
hepatocellular carcinoma
ICER
incremental cost-effectiveness ratio 
MRE magnetic resonance elastography

NAFLD nonalcoholic fatty liver disease

NASH nonalcoholic steatohepatitis

QALY quality-adjusted life year

TE transient elastography

\section{References}

1. Neuschwander-Tetri BA, Caldwell SH. Nonalcoholic steatohepatitis: summary of an AASLD Single Topic Conference. Hepatology. 2003; 37:1202-1219. [PubMed: 12717402]

2. Bellentani S, Scaglioni F, Marino M, Bedogni G. Epidemiology of non-alcoholic fatty liver disease. Dig Dis. 2010; 28:155-161. [PubMed: 20460905]

3. Adams LA, Sanderson S, Lindor KD, Angulo P. The histological course of nonalcoholic fatty liver disease: a longitudinal study of 103 patients with sequential liver biopsies. J Hepatol. 2005; 42:132138. [PubMed: 15629518]

4. Browning JD, Szczepaniak LS, Dobbins R, et al. Prevalence of hepatic steatosis in an urban population in the United States: impact of ethnicity. Hepatology. 2004; 40:1387-1395. [PubMed: 15565570]

5. Farrell GC, Larter CZ. Nonalcoholic fatty liver disease: from steatosis to cirrhosis. Hepatology. 2006; 43:S99-S112. [PubMed: 16447287]

6. Ekstedt M, Franzen LE, Mathiesen UL, et al. Long-term follow-up of patients with NAFLD and elevated liver enzymes. Hepatology. 2006; 44:865-873. [PubMed: 17006923]

7. Ascha MS, Hanouneh IA, Lopez R, Tamimi TA, Feldstein AF, Zein NN. The incidence and risk factors of hepatocellular carcinoma in patients with nonalcoholic steatohepatitis. Hepatology. 2010; 51:1972-1978. [PubMed: 20209604]

8. Ratziu V, Bonyhay L, Di Martino V, et al. Survival, liver failure, and hepatocellular carcinoma in obesity-related cryptogenic cirrhosis. Hepatology. 2002; 35:1485-1493. [PubMed: 12029634]

9. Starley BQ, Calcagno CJ, Harrison SA. Nonalcoholic fatty liver disease and hepatocellular carcinoma: a weighty connection. Hepatology. 2010; 51:1820-1832. [PubMed: 20432259]

10. Charlton MR, Burns JM, Pedersen RA, Watt KD, Heimbach JK, Dierkhising RA. Frequency and outcomes of liver transplantation for nonalcoholic steatohepatitis in the United States. Gastroenterology. 2011; 141:1249-1253. [PubMed: 21726509]

11. Ratziu V, Bellentani S, Cortez-Pinto H, Day C, Marchesini G. A position statement on NAFLD/ NASH based on the EASL 2009 special conference. J Hepatol. 2010; 53:372-384. [PubMed: 20494470]

12. Chalasani N, Younossi Z, Lavine JE, et al. The diagnosis and management of non-alcoholic fatty liver disease: practice Guideline by the American Association for the Study of Liver Diseases, American College of Gastroenterology, and the American Gastroenterological Association. Hepatology. 2012; 55:2005-2023. [PubMed: 22488764]

13. Rockey DC, Caldwell SH, Goodman ZD, Nelson RC, Smith AD. American Association for the Study of Liver D. Liver biopsy. Hepatology. 2009; 49:1017-1044. [PubMed: 19243014]

14. Musso G, Gambino R, Cassader M, Pagano G. Meta-analysis: natural history of non-alcoholic fatty liver disease (NAFLD) and diagnostic accuracy of non-invasive tests for liver disease severity. Ann Med. 2011; 43:617-649. [PubMed: 21039302]

15. Talwalkar JA, Kurtz DM, Schoenleber SJ, West CP, Montori VM. Ultrasound-based transient elastography for the detection of hepatic fibrosis: systematic review and meta-analysis. Clin Gastroenterol Hepatol. 2007; 5:1214-1220. [PubMed: 17916549]

16. Machado MV, Cortez-Pinto H. Non-invasive diagnosis of non-alcoholic fatty liver disease. A critical appraisal. J Hepatol. 2013; 58:1007-1019. [PubMed: 23183525] 
17. Castera L, Pinzani M. Biopsy and non-invasive methods for the diagnosis of liver fibrosis: does it take two to tango? Gut. 2010; 59:861-866. [PubMed: 20581229]

18. Promrat K, Kleiner DE, Niemeier HM, et al. Randomized controlled trial testing the effects of weight loss on nonalcoholic steatohepatitis. Hepatology. 2010; 51:121-129. [PubMed: 19827166]

19. Sanyal AJ, Chalasani N, Kowdley KV, et al. Pioglitazone, vitamin E, or placebo for nonalcoholic steatohepatitis. N Engl J Med. 2010; 362:1675-1685. [PubMed: 20427778]

20. Mahady SE, Webster AC, Walker S, Sanyal A, George J. The role of thiazolidinediones in nonalcoholic steatohepatitis - a systematic review and meta analysis. J Hepatol. 2011; 55:1383-1390. [PubMed: 21703200]

21. Mahady SE, Wong G, Craig JC, George J. Pioglitazone and vitamin E for nonalcoholic steatohepatitis: a cost utility analysis. Hepatology. 2012; 56:2172-2179. [PubMed: 22707355]

22. Petrou S, Gray A. Economic evaluation using decision analytical modelling: design, conduct, analysis, and reporting. BMJ. 2011; 342:d1766. [PubMed: 21482590]

23. Hashimoto E, Yatsuji S, Tobari M, et al. Hepatocellular carcinoma in patients with nonalcoholic steatohepatitis. J Gastroenterol. 2009; 44(Suppl 19):89-95. [PubMed: 19148800]

24. Vernon G, Baranova A, Younossi ZM. Systematic review: the epidemiology and natural history of non-alcoholic fatty liver disease and non-alcoholic steatohepatitis in adults. Aliment Pharmacol Ther. 2011; 34:274-285. [PubMed: 21623852]

25. Canadian Agency for Drugs and Technologies in Health. Guidelines for the economic evaluation of health technologies: Canada. 3. Ottawa: 2006. p. 75

26. Wang QB, Zhu H, Liu HL, Zhang B. Performance of magnetic resonance elastography and diffusion-weighted imaging for the staging of hepatic fibrosis: A meta-analysis. Hepatology. 2012; 56:239-247. [PubMed: 22278368]

27. Friedrich-Rust M, Romen D, Vermehren J, et al. Acoustic radiation force impulse-imaging and transient elastography for non-invasive assessment of liver fibrosis and steatosis in NAFLD. Eur J Radiol. 2011; doi: 10.1016/j.ejrad.2011.10.029

28. West J, Card TR. Reduced mortality rates following elective percutaneous liver biopsies. Gastroenterology. 2010; 139:1230-1237. [PubMed: 20547160]

29. Nascimbeni F, Pais R, Bellentani S, et al. From NAFLD in clinical practice to answers from guidelines. J Hepatol. 2013; doi: 10.1016/j.jhep.2013.05.044

30. Chalasani NP, Sanyal AJ, Kowdley KV, et al. Pioglitazone versus vitamin E versus placebo for the treatment of non-diabetic patients with non-alcoholic steatohepatitis: PIVENS trial design. Contemp Clin Trials. 2009; 30:88-96. [PubMed: 18804555]

31. Miller DK, Homan SM. Determining transition probabilities: confusion and suggestions. Med Decis Making. 1994; 14:52-58. [PubMed: 8152357]

32. Canadian Medical Association. Canadian Provincial Billing Guide. Health Canada, Government of Canada; Available at http://www.cma.ca/practicemanagement/medical-billing [Accessed July, 2013]

33. Steadman, RLL., Lorenzetti, D., Noseworthy, T., Rose, S., Sutherland, L., Clement, F. Unit UoCHTA. A Health Technology Assessment of Transient Elastography in Liver Disease. Alberta Health Technologies Decision Process; Alberta: 2012.

34. Barry, MH. Health NSDo. Nova Scotia. 2004. Magnetic Resonance Imaging Needs Assessment; p. 38

35. Canadian Institute for Health Information. Medical Imaging in Canada, 2007. Ottawa, Ont: CIHI; 2008.

36. Peviva. [Accessed July, 2013] M30 Apoptosense ELISA. Available at http://www.peviva.se/m30apoptosense-elisa_us.aspx

37. Canadian Institute for Health Information. The Cost of Acute Care Hospital Stays by Medical Condition in Canada, 2004-2005. Ottawa: CIHI; 2008.

38. Thein HH, Isaranuwatchai W, Campitelli MA, et al. Health care costs associated with hepatocellular carcinoma: a population-based study. Hepatology. 2013; 58:1375-1384. [PubMed: 23300063] 
39. Taylor MC, Greig PD, Detsky AS, McLeod RS, Abdoh A, Krahn MD. Factors associated with the high cost of liver transplantation in adults. Can J Surg. 2002; 45:425-434. [PubMed: 12500917]

40. Bank of Canada. [Accessed July, 2013] Inflation Calculator. Available at http:// www.bankofcanada.ca/rates/related/inflation-calculator

41. David K, Kowdley KV, Unalp A, et al. Quality of life in adults with nonalcoholic fatty liver disease: baseline data from the nonalcoholic steatohepatitis clinical research network. Hepatology. 2009; 49:1904-1912. [PubMed: 19434741]

42. Nichol MB, Sengupta N, Globe DR. Evaluating quality-adjusted life years: estimation of the health utility index (HUI2) from the SF-36. Med Decis Making. 2001; 21:105-112. [PubMed: 11310943]

43. Chong CA, Gulamhussein A, Heathcote EJ, et al. Health-state utilities and quality of life in hepatitis C patients. Am J Gastroenterol. 2003; 98:630-638. [PubMed: 12650799]

44. Younossi ZM, Boparai N, McCormick M, Price LL, Guyatt G. Assessment of utilities and healthrelated quality of life in patients with chronic liver disease. Am J Gastroenterol. 2001; 96:579-583. [PubMed: 11232711]

45. Ratcliffe J, Longworth L, Young T, et al. Assessing health-related quality of life pre- and post-liver transplantation: a prospective multicenter study. Liver Transpl. 2002; 8:263-270. [PubMed: 11910572]

46. McLernon DJ, Dillon J, Donnan PT. Health-state utilities in liver disease: a systematic review. Med Decis Making. 2008; 28:582-592. [PubMed: 18424560]

47. Yoneda M, Suzuki K, Kato S, et al. Nonalcoholic fatty liver disease: US-based acoustic radiation force impulse elastography. Radiology. 2010; 256:640-647. [PubMed: 20529989]

48. Bota S, Herkner H, Sporea I, et al. Meta-analysis: ARFI elastography versus transient elastography for the evaluation of liver fibrosis. Liver Int. 2013; 33:1138-1147. [PubMed: 23859217]

49. Sasso M, Beaugrand M, de Ledinghen V, et al. Controlled attenuation parameter (CAP): a novel VCTE guided ultrasonic attenuation measurement for the evaluation of hepatic steatosis: preliminary study and validation in a cohort of patients with chronic liver disease from various causes. Ultrasound Med Biol. 2010; 36:1825-1835. [PubMed: 20870345]

50. Sasso M, Miette V, Sandrin L, Beaugrand M. The controlled attenuation parameter (CAP): a novel tool for the non-invasive evaluation of steatosis using Fibroscan. Clin Res Hepatol Gastroenterol. 2012; 36:13-20. [PubMed: 21920839]

51. Myers RP, Pomier-Layrargues G, Kirsch R, et al. Feasibility and diagnostic performance of the FibroScan XL probe for liver stiffness measurement in overweight and obese patients. Hepatology. 2012; 55:199-208. [PubMed: 21898479]

52. de Ledinghen V, Vergniol J, Foucher J, El-Hajbi F, Merrouche W, Rigalleau V. Feasibility of liver transient elastography with FibroScan using a new probe for obese patients. Liver Int. 2010; 30:1043-1048. [PubMed: 20492500]

53. Friedrich-Rust M, Romen D, Vermehren J, et al. Acoustic radiation force impulse-imaging and transient elastography for non-invasive assessment of liver fibrosis and steatosis in NAFLD. Eur J Radiol. 2012; 81:e325-331. [PubMed: 22119555]

54. Palmeri ML, Wang MH, Rouze NC, et al. Noninvasive evaluation of hepatic fibrosis using acoustic radiation force-based shear stiffness in patients with nonalcoholic fatty liver disease. J Hepatol. 2011; 55:666-672. [PubMed: 21256907]

55. Guzman-Aroca F, Frutos-Bernal MD, Bas A, et al. Detection of non-alcoholic steatohepatitis in patients with morbid obesity before bariatric surgery: preliminary evaluation with acoustic radiation force impulse imaging. Eur Radiol. 2012; 22:2525-2532. [PubMed: 22648049]

56. Moynihan R, Doust J, Henry D. Preventing overdiagnosis: how to stop harming the healthy. BMJ. 2012; 344:e3502. [PubMed: 22645185]

57. Argo CK, Caldwell SH. Epidemiology and natural history of non-alcoholic steatohepatitis. Clin Liver Dis. 2009; 13:511-531. [PubMed: 19818302]

58. Pais R, Pascale A, Fedchuck L, Charlotte F, Poynard T, Ratziu V. Progression from isolated steatosis to steatohepatitis and fibrosis in nonalcoholic fatty liver disease. Gastroenterol Clin Biol. 2010; doi: 10.1016/j.gcb.2010.06.004

59. Angulo P. Long-term mortality in nonalcoholic fatty liver disease: is liver histology of any prognostic significance? Hepatology. 2010; 51:373-375. [PubMed: 20101746] 
60. Boursier J, Chaigneau J, Roullier V, et al. Steatosis degree, measured by morphometry, is linked to other liver lesions and metabolic syndrome components in patients with NAFLD. Eur J Gastroenterol Hepatol. 2011; 23:974-981. [PubMed: 21904208]

61. Permutt ZLT, Peterson MR, et al. Correlation between liver histology and novel magnetic resonance imaging in adult patients with non-alcoholic fatty liver disease - MRI accurately quantifies hepatic steatosis in NAFLD. Alimentary pharmacology \& therapeutics. 2012; 36(1):229. [PubMed: 22554256]

62. Chen J, Zhu Y, Zheng Q, Jiang J. Serum cytokeratin-18 in the diagnosis of non-alcoholic steatohepatitis: A meta-analysis. Hepatol Res. 2013; doi: 10.1111/hepr.12197

63. Chen J, Talwalkar JA, Yin M, Glaser KJ, Sanderson SO, Ehman RL. Early detection of nonalcoholic steatohepatitis in patients with nonalcoholic fatty liver disease by using MR elastography. Radiology. 2011; 259:749-756. [PubMed: 21460032]

64. Abner EL, Schmitt FA, Mendiondo MS, Marcum JL, Kryscio RJ. Vitamin E and all-cause mortality: a meta-analysis. Curr Aging Sci. 2011; 4:158-170. [PubMed: 21235492]

65. Miller ER 3rd, Pastor-Barriuso R, Dalal D, Riemersma RA, Appel LJ, Guallar E. Meta-analysis: high-dosage vitamin E supplementation may increase all-cause mortality. Ann Intern Med. 2005; 142:37-46. [PubMed: 15537682]

66. Mamtani R, Haynes K, Bilker WB, et al. Association between longer therapy with thiazolidinediones and risk of bladder cancer: a cohort study. J Natl Cancer Inst. 2012; 104:14111421. [PubMed: 22878886]

67. Vallarino C, Perez A, Fusco G, et al. Comparing pioglitazone to insulin with respect to cancer, cardiovascular and bone fracture endpoints, using propensity score weights. Clin Drug Investig. 2013; 33:621-631.

68. Monami M, Dicembrini I, Mannucci E. Thiazolidinediones and cancer: results of a meta-analysis of randomized clinical trials. Acta Diabetol. 2014; 51:91-101. [PubMed: 23851465]

69. Lazo M, Hernaez R, Eberhardt MS, et al. Prevalence of nonalcoholic fatty liver disease in the United States: the Third National Health and Nutrition Examination Survey, 1988-1994. Am J Epidemiol. 2013; 178:38-45. [PubMed: 23703888]

70. Targher G, Bertolini L, Padovani R, et al. Prevalence of nonalcoholic fatty liver disease and its association with cardiovascular disease among type 2 diabetic patients. Diabetes Care. 2007; 30:1212-1218. [PubMed: 17277038]

71. Angulo P. Nonalcoholic fatty liver disease. N Engl J Med. 2002; 346:1221-1231. [PubMed: 11961152]

72. Medina J, Fernandez-Salazar LI, Garcia-Buey L, Moreno-Otero R. Approach to the pathogenesis and treatment of nonalcoholic steatohepatitis. Diabetes Care. 2004; 27:2057-2066. [PubMed: 15277442]

73. Preiss D, Sattar N. Non-alcoholic fatty liver disease: an overview of prevalence, diagnosis, pathogenesis and treatment considerations. Clin Sci (Lond). 2008; 115:141-150. [PubMed: 18662168]

74. Williams CD, Stengel J, Asike MI, et al. Prevalence of nonalcoholic fatty liver disease and nonalcoholic steatohepatitis among a largely middle-aged population utilizing ultrasound and liver biopsy: a prospective study. Gastroenterology. 2011; 140:124-131. [PubMed: 20858492]

75. Wanless IR, Lentz JS. Fatty liver hepatitis (steatohepatitis) and obesity: an autopsy study with analysis of risk factors. Hepatology. 1990; 12:1106-1110. [PubMed: 2227807]

76. Silverman JF, Pories WJ, Caro JF. Liver pathology in diabetes mellitus and morbid obesity. Clinical, pathological, and biochemical considerations. Pathol Annu. 1989; 24(Pt 1):275-302. [PubMed: 2654841]

77. Pinto HC, Baptista A, Camilo ME, Valente A, Saragoca A, de Moura MC. Nonalcoholic steatohepatitis. Clinicopathological comparison with alcoholic hepatitis in ambulatory and hospitalized patients. Dig Dis Sci. 1996; 41:172-179. [PubMed: 8565753]

78. World Gastroenterology Organisation. WGO Global Guidelines Nonalcoholic Fatty Liver Disease and Nonalcoholic Steatohepatitis. 2012. p. 29 
79. Zelber-Sagi S, Lotan R, Shlomai A, et al. Predictors for incidence and remission of NAFLD in the general population during a seven-year prospective follow-up. J Hepatol. 2012; 56:1145-1151. [PubMed: 22245895]

80. Teli MR, James OF, Burt AD, Bennett MK, Day CP. The natural history of nonalcoholic fatty liver: a follow-up study. Hepatology. 1995; 22:1714-1719. [PubMed: 7489979]

81. Wong VW, Wong GL, Choi PC, et al. Disease progression of non-alcoholic fatty liver disease: a prospective study with paired liver biopsies at 3 years. Gut. 2010; 59:969-974. [PubMed: 20581244]

82. Rafiq N, Bai C, Fang Y, et al. Long-term follow-up of patients with nonalcoholic fatty liver. Clin Gastroenterol Hepatol. 2009; 7:234-238. [PubMed: 19049831]

83. Fassio E, Alvarez E, Dominguez N, Landeira G, Longo C. Natural history of nonalcoholic steatohepatitis: a longitudinal study of repeat liver biopsies. Hepatology. 2004; 40:820-826. [PubMed: 15382171]

84. Hui AY, Wong VW, Chan HL, et al. Histological progression of non-alcoholic fatty liver disease in Chinese patients. Aliment Pharmacol Ther. 2005; 21:407-413. [PubMed: 15709991]

85. Bhala N, Angulo P, van der Poorten D, et al. The natural history of nonalcoholic fatty liver disease with advanced fibrosis or cirrhosis: an international collaborative study. Hepatology. 2011; 54:1208-1216. [PubMed: 21688282]

86. Hui JM, Kench JG, Chitturi S, et al. Long-term outcomes of cirrhosis in nonalcoholic steatohepatitis compared with hepatitis C. Hepatology. 2003; 38:420-427. [PubMed: 12883486]

87. Sanyal AJ, Banas C, Sargeant C, et al. Similarities and differences in outcomes of cirrhosis due to nonalcoholic steatohepatitis and hepatitis C. Hepatology. 2006; 43:682-689. [PubMed: 16502396]

88. Soderberg C, Stal P, Askling J, et al. Decreased survival of subjects with elevated liver function tests during a 28-year follow-up. Hepatology. 2010; 51:595-602. [PubMed: 20014114]

89. McCullough AJ. The clinical features, diagnosis and natural history of nonalcoholic fatty liver disease. Clin Liver Dis. 2004; 8:521-533. viii. [PubMed: 15331061]

90. Marrero JA, Fontana RJ, Barrat A, et al. Prognosis of hepatocellular carcinoma: comparison of 7 staging systems in an American cohort. Hepatology. 2005; 41:707-716. [PubMed: 15795889]

91. Altekruse SF, McGlynn KA, Reichman ME. Hepatocellular carcinoma incidence, mortality, and survival trends in the United States from 1975 to 2005. J Clin Oncol. 2009; 27:1485-1491. [PubMed: 19224838]

92. Greten TF, Papendorf F, Bleck JS, et al. Survival rate in patients with hepatocellular carcinoma: a retrospective analysis of 389 patients. Br J Cancer. 2005; 92:1862-1868. [PubMed: 15870713]

93. Lang H, Sotiropoulos GC, Brokalaki EI, et al. Survival and recurrence rates after resection for hepatocellular carcinoma in noncirrhotic livers. J Am Coll Surg. 2007; 205:27-36. [PubMed: 17617329]

94. Perry JF, Charlton B, Koorey DJ, et al. Outcome of patients with hepatocellular carcinoma referred to a tertiary centre with availability of multiple treatment options including cadaveric liver transplantation. Liver Int. 2007; 27:1240-1248. [PubMed: 17919236]

95. Feldstein AE, Wieckowska A, Lopez AR, Liu YC, Zein NN, McCullough AJ. Cytokeratin-18 fragment levels as noninvasive biomarkers for nonalcoholic steatohepatitis: a multicenter validation study. Hepatology. 2009; 50:1072-1078. [PubMed: 19585618]

96. Angulo P, Hui JM, Marchesini G, et al. The NAFLD fibrosis score: a noninvasive system that identifies liver fibrosis in patients with NAFLD. Hepatology. 2007; 45:846-854. [PubMed: 17393509]

97. Friedrich-Rust M, Ong MF, Martens S, et al. Performance of transient elastography for the staging of liver fibrosis: a meta-analysis. Gastroenterology. 2008; 134:960-974. [PubMed: 18395077]

98. Kwok R, Tse YK, Wong GL, et al. Systematic review with meta-analysis: non-invasive assessment of non-alcoholic fatty liver disease--the role of transient elastography and plasma cytokeratin-18 fragments. Aliment Pharmacol Ther. 2014; 39:254-269. [PubMed: 24308774]

99. Friedrich-Rust M, Nierhoff J, Lupsor M, et al. Performance of Acoustic Radiation Force Impulse imaging for the staging of liver fibrosis: a pooled meta-analysis. J Viral Hepat. 2012; 19:e212-219. [PubMed: 22239521] 
100. Nierhoff J, Chavez Ortiz AA, Herrmann E, Zeuzem S, Friedrich-Rust M. The efficiency of acoustic radiation force impulse imaging for the staging of liver fibrosis: a meta-analysis. Eur Radiol. 2013; 23:3040-3053. [PubMed: 23801420]

101. Castera L, Foucher J, Bernard PH, et al. Pitfalls of liver stiffness measurement: a 5-year prospective study of 13,369 examinations. Hepatology. 2010; 51:828-835. [PubMed: 20063276]

102. Murtagh J, Foerster V. Transient elastography (FibroScan) for non-invasive assessment of liver fibrosis. Issues Emerg Health Technol. 2006:1-4.

103. Carlson JJ, Kowdley KV, Sullivan SD, Ramsey SD, Veenstra DL. An evaluation of the potential cost-effectiveness of non-invasive testing strategies in the diagnosis of significant liver fibrosis. J Gastroenterol Hepatol. 2009; 24:786-791. [PubMed: 19457153]

104. DeKoven, M. Memo to GE Healthcare from IMS Health Incorporated. 2010. Cost comparison: liver biopsy versus abdominal MRI.

105. Myers RP, Fong A, Shaheen AA. Utilization rates, complications and costs of percutaneous liver biopsy: a population-based study including 4275 biopsies. Liver Int. 2008; 28:705-712. [PubMed: 18433397]

106. Régie de l'assurance maladie du Québec (RAMQ). RAMQ liste de medicaments 2013. Quebec Ministry of Health and Social Services; 2013. Available at https://http:// www.prod.ramq.gouv.qc.ca/DPI/PO/Commun/PDF/Liste_Med/Liste_Med/ liste_med_cor_2013_07_15_fr.pdf [Accessed August, 2013]

107. Thompson Coon J, Rogers G, Hewson P, et al. Surveillance of cirrhosis for hepatocellular carcinoma: a cost-utility analysis. Br J Cancer. 2008; 98:1166-1175. [PubMed: 18382459]

108. Aberg F, Maklin S, Rasanen P, et al. Cost of a quality-adjusted life year in liver transplantation: the influence of the indication and the model for end-stage liver disease score. Liver Transpl. 2011; 17:1333-1343. [PubMed: 21770017] 


\section{KEY POINTS}

1. This cost-utility analysis suggests that screening for nonalcoholic steatohepatitis may be cost-effective.

2. In particular, screening of high-risk obese or diabetic populations is more cost-effective.

3. Magnetic resonance elastography was more cost-effective to confirm disease compared to biopsy.

4. More studies are needed to determine quality of life in nonalcoholic steatohepatitis.

5. More management strategies for nonalcoholic steatohepatitis are also needed. 


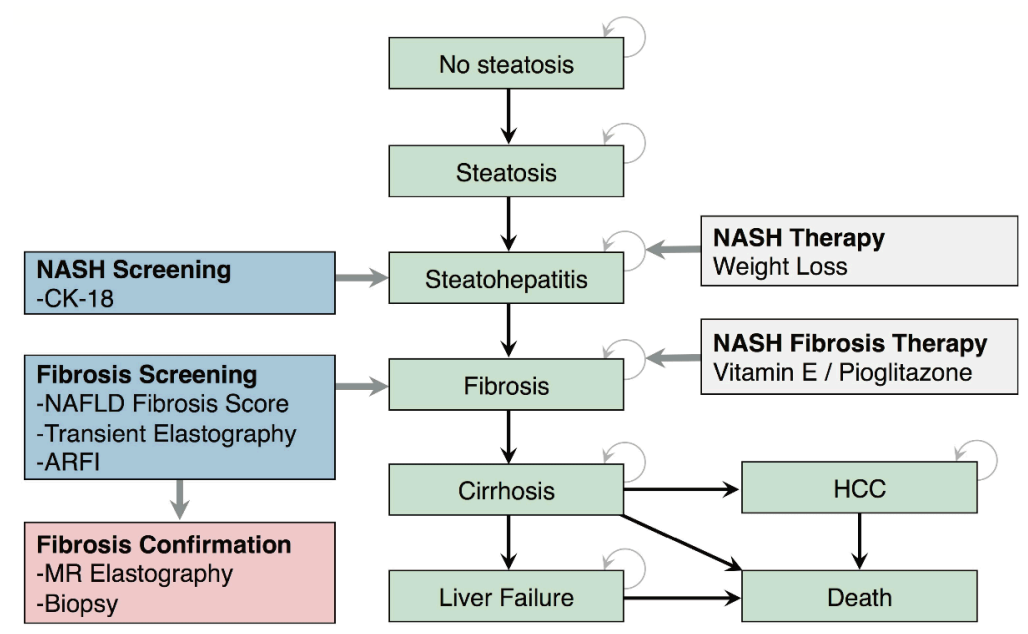

Figure 1.

Markov model illustrating the natural history of NAFLD, screening strategies, and therapies. 
A

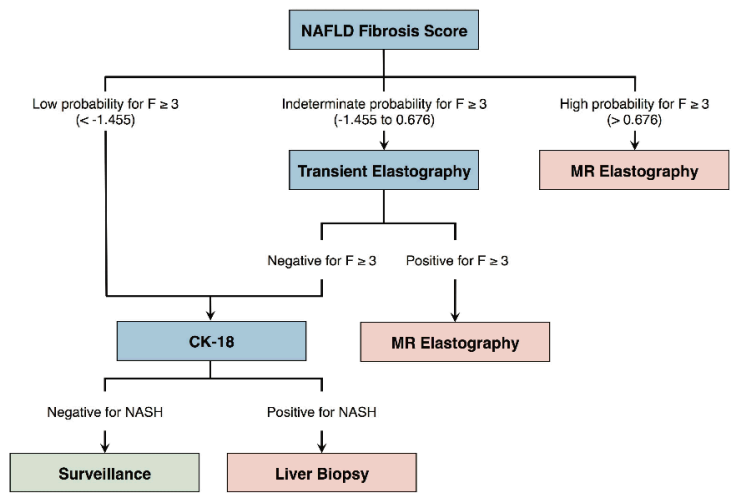

Eur Radiol. Author manuscript; available in PMC 2016 April 19. 


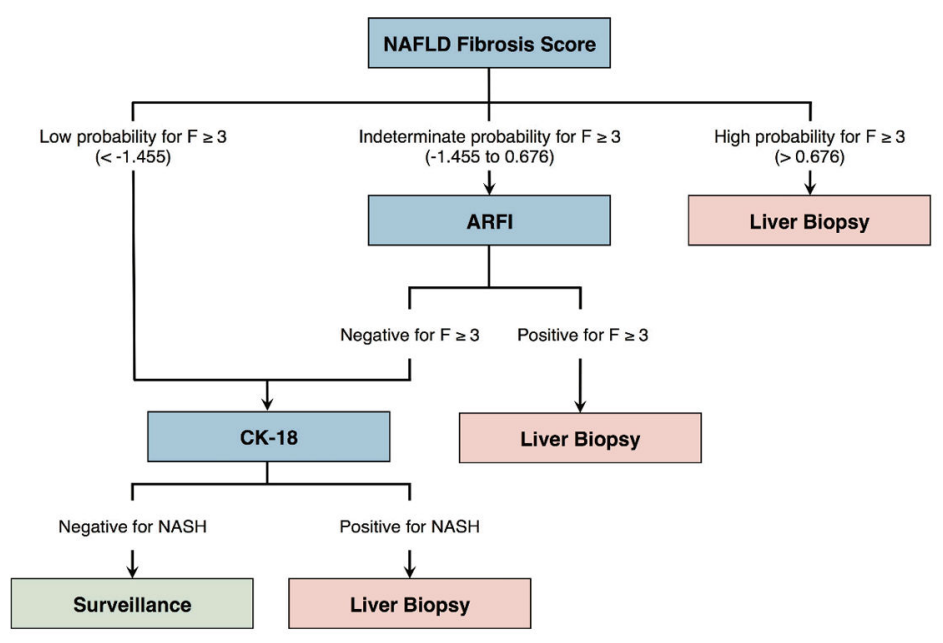

$\mathrm{C}$

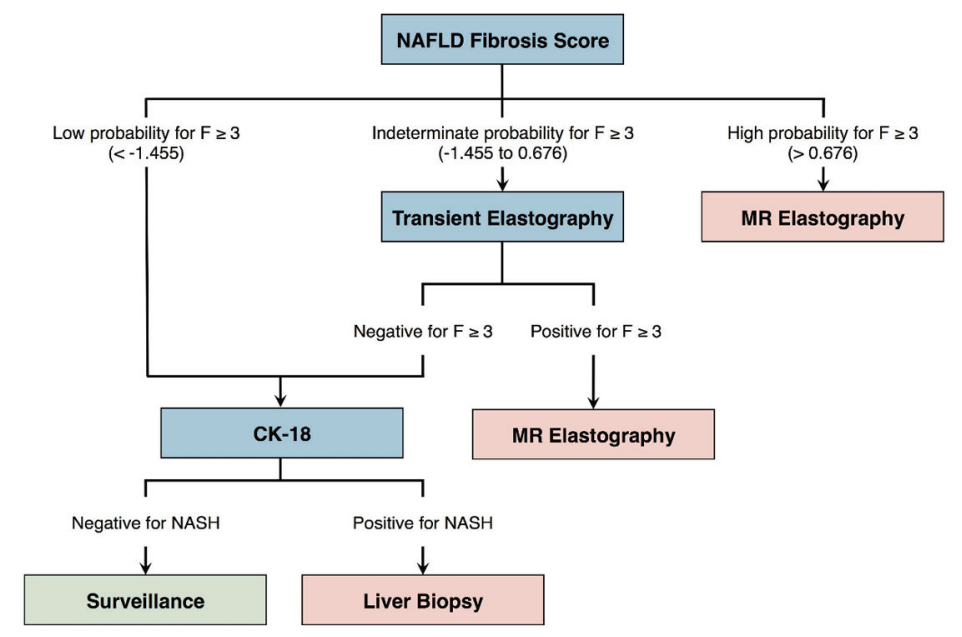

Figure 2. Decision trees illustrating the 3 NASH screening algorithms investigated in cost-utility analysis

(A) Sequential algorithm with NAFLD fibrosis score/TE/CK-18 with liver biopsy confirmation. (B) Sequential algorithm with NAFLD fibrosis score/ARFI/CK-18 with liver biopsy confirmation. (C) Sequential algorithm with NAFLD fibrosis score/TE/CK-18 with MRE confirmation. 


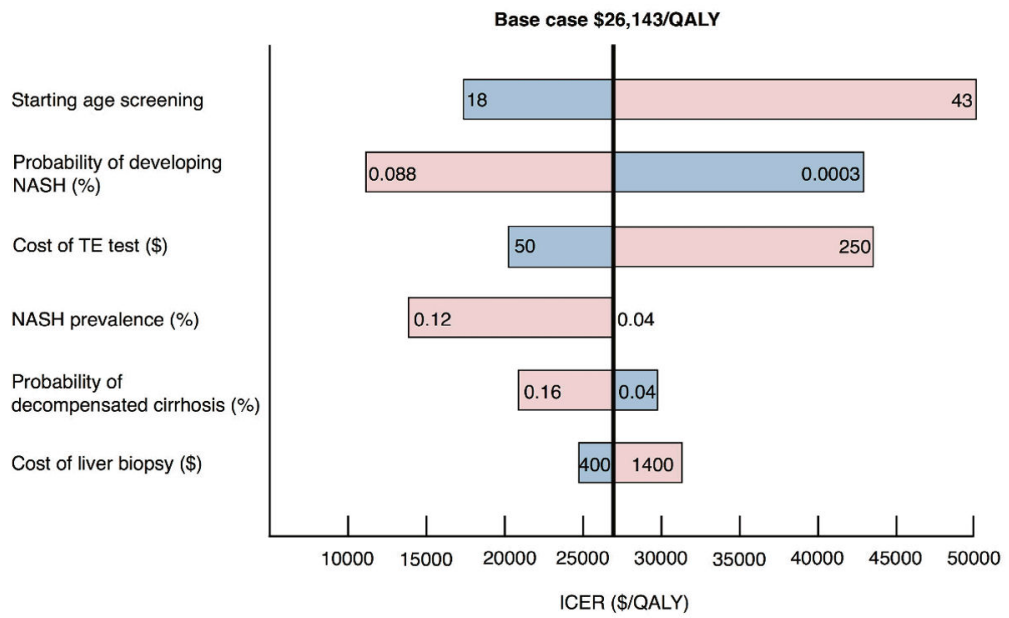

Figure 3.

One-way sensitivity analysis for the NAFLD fibrosis score/TE/CK-18 sequential strategy with MRE confirmation and vitamin $\mathrm{E}$ as treatment.

Abbreviations: NAFLD, nonalcoholic fatty liver disease; NASH, nonalcoholic steatohepatitis; TE, transient elastography; CK-18, cytokeratin-18; ARFI, acoustic radiation force impulse; MRE, magnetic resonance elastography. 


\section{Table 1}

\section{Model Parameter}

\begin{tabular}{|c|c|c|}
\hline Parameters & Base Estimate (Range) & References \\
\hline \multicolumn{3}{|l|}{ Prevalence } \\
\hline Prevalence of steatosis in general population & $0.23(0.16-0.30)$ & {$[4 ; 5 ; 69]$} \\
\hline Prevalence of steatosis in type 2 diabetes population & 0.70 & [70] \\
\hline Prevalence of steatosis in obese population & $0.75(0.64-0.90)$ & $\begin{array}{c}{[71]} \\
{[5 ; 72 ; 73]}\end{array}$ \\
\hline Prevalence of NASH in general population & $0.04(0.02-0.122)$ & {$[2 ; 24 ; 74 ; 75]$} \\
\hline Prevalence of NASH in type 2 diabetes population & $0.25(0.25-0.30)$ & {$[14 ; 76]$} \\
\hline Prevalence of NASH in obese population & $0.20(0.19-0.50)$ & {$[5 ; 71-73]$} \\
\hline Prevalence of NASH-cirrhosis in general population & $0.0019(0.0018-0.0020)$ & {$[77 ; 78]$} \\
\hline Prevalence of NASH-cirrhosis in type 2 diabetes population & $0.02(0.02-0.03)$ & Author's assumptions \\
\hline Prevalence of NASH-cirrhosis in obese population & $0.02(0.02-0.03)$ & {$[72]$} \\
\hline \multicolumn{3}{|l|}{ Annual transition probabilities } \\
\hline Probability of developing steatosis & $0.029(0.02-0.04)$ & {$[24 ; 79]$} \\
\hline Probability of developing NASH & $0.0084(0.00029-0.088)$ & {$[24 ; 58 ; 80 ; 81]$} \\
\hline Probability of NASH liver-related mortality & $0.0038(0.002-0.01)$ & {$[6 ; 59 ; 82]$} \\
\hline Probability of developing fibrosis & $0.089(0.065-0.092)$ & {$[3 ; 24 ; 57 ; 83]$} \\
\hline Probability of worsening fibrosis & $0.11(0.10-0.13)$ & {$[3 ; 24 ; 81 ; 84]$} \\
\hline Probability of developing cirrhosis & $0.02-0.06$ & {$[6 ; 85]$} \\
\hline Probability of NASH-cirrhosis liver-related mortality & $0.034(0.015-0.049)$ & {$[59 ; 86-88]$} \\
\hline Probability of developing decompensated cirrhosis & $0.06(0.04-0.16)$ & {$[6 ; 11 ; 57 ; 86 ; 87]$} \\
\hline Probability of decompensated cirrhosis-related mortality & $0.16(0.15-0.38)$ & {$[8 ; 89]$} \\
\hline Probability of developing HCC & $0.029(0.017-0.08)$ & {$[7 ; 8 ; 21 ; 23 ; 82 ; 87]$} \\
\hline Probability of hepatoma mortality at year 1 & $0.52(0.47-0.58)$ & [90-92] \\
\hline Probability of hepatoma mortality in subsequent years & $0.068(0.068-0.23)$ & {$[92 ; 93]$} \\
\hline Probability of liver transplantation & $0.05(0.05-0.25)$ & {$[21 ; 94]$} \\
\hline \multicolumn{3}{|l|}{ Sensitivity for NASH detection } \\
\hline Plasma cytokeratin- 18 fragments & $0.77(0.64-0.92)$ & {$[14 ; 62 ; 95]$} \\
\hline \multicolumn{3}{|l|}{ Sensitivity for advanced fibrosis ( $\geq \mathbf{F 3}$ ) } \\
\hline NAFLD fibrosis score & $0.64(0.59-0.70)$ & {$[14 ; 96]$} \\
\hline Transient elastography (TE) & $0.85(0.58-0.95)$ & {$[14 ; 15 ; 48 ; 97 ; 98]$} \\
\hline Acoustic radiation force impulse (ARFI) & $0.89(0.87-0.99)$ & {$[47 ; 48 ; 99 ; 100]$} \\
\hline Magnetic resonance elastography (MRE) & $0.92(0.85-0.96)$ & [26] \\
\hline \multicolumn{3}{|l|}{ Technical failure of elastography methods } \\
\hline Rate of technical failure of TE & 0.16 & [101] \\
\hline Rate of technical failure of ARFI & 0.021 & [48] \\
\hline \multicolumn{3}{|l|}{ Treatment response } \\
\hline Histological improvement to lifestyle changes & 2.40 & [18] \\
\hline
\end{tabular}




\begin{tabular}{lcc}
\hline Parameters & Base Estimate (Range) & References \\
\hline Histological improvement to pioglitazone & $1.38(1.01-1.89)$ & {$[19 ; 20]$} \\
\hline Histological improvement to vitamin E & $1.35(0.87-2.09)$ & {$[19 ; 20]$} \\
\hline Complications of liver biopsy & & \\
\hline Rate of mortality & 0.002 & {$[28]$} \\
\hline Rate of major bleeding & 0.0065 & {$[28]$} \\
\hline
\end{tabular}

Abbreviations: NASH, nonalcoholic steatohepatitis; HCC, hepatocellular carcinoma; NAFLD, nonalcoholic fatty liver disease. 
Table 2

\section{Health Care Costs (CAN\$, Canadian Dollars)}

\begin{tabular}{|c|c|c|}
\hline Parameters & Base Estimate (Range) & References \\
\hline \multicolumn{3}{|l|}{ Annual clinical care costs } \\
\hline No care & 77.20 & {$[32]$} \\
\hline Routine care and lifestyle changes & 325.00 & {$[32]$} \\
\hline Routine care and pioglitazone & $2,106.20$ & {$[32]$} \\
\hline Routine care and vitamin $\mathrm{E}$ & 463.70 & {$[32]$} \\
\hline Compensated cirrhosis and pioglitazone & $2,183.40$ & {$[32]$} \\
\hline Compensated cirrhosis and vitamin $\mathrm{E}$ & 540.90 & [32] \\
\hline Decompensated cirrhosis & $16,679.50(10,884-22,475)$ & {$[32 ; 37]$} \\
\hline Hepatocellular carcinoma (net over 5 years) & $15,949.80$ & {$[38]$} \\
\hline Liver transplant (1st year) & $163,818.77$ & {$[39]$} \\
\hline \multicolumn{3}{|l|}{ Itemized clinical care } \\
\hline Specialist consultation (initial) & 157.00 & {$[32]$} \\
\hline Specialist consultation (follow-up) & 105.25 & {$[32]$} \\
\hline Primary care doctor consultation & 77.20 & {$[32]$} \\
\hline Dietitian/counselling & 62.75 & {$[32]$} \\
\hline \multicolumn{3}{|l|}{ Laboratory } \\
\hline Full blood count & 11.03 & {$[32]$} \\
\hline Liver function tests & 20.70 & {$[32]$} \\
\hline Lipids & 21.31 & {$[32]$} \\
\hline Oral glucose tolerance test & 15.68 & {$[32]$} \\
\hline Hepatitis $\mathrm{C}$ antibody & 27.24 & {$[32]$} \\
\hline Hepatitis B surface antigen & 36.30 & {$[32]$} \\
\hline Anti nuclear antibody & 27.24 & {$[32]$} \\
\hline \multicolumn{3}{|l|}{ Screening methods } \\
\hline NAFLD fibrosis score & 12.95 & {$[32]$} \\
\hline Plasma cytokeratin-18 fragments (CK-18) & 6.44 & {$[36]$} \\
\hline Transient elastography (TE) & 99.44 & {$[33 ; 35]$} \\
\hline Ultrasound-based elastography (ARFI) & 114.62 & {$[33 ; 35]$} \\
\hline \multicolumn{3}{|l|}{ Diagnostic method } \\
\hline Magnetic resonance elastography & $333.98(250-400)$ & {$[34 ; 35]$} \\
\hline Liver biopsy & $595.60(450-1300)$ & {$[102-104]$} \\
\hline \multicolumn{3}{|l|}{ Complications } \\
\hline Post-biopsy complication requiring hospitalization & 4,579 & {$[105]$} \\
\hline \multicolumn{3}{|l|}{ Treatment (yearly) } \\
\hline Pioglitazone (Actos) & $1,084.05$ & {$[106]$} \\
\hline Vitamin E (800 IU) & 138.7 & {$[106]$} \\
\hline
\end{tabular}

Abbreviations: NAFLD, nonalcoholic fatty liver disease. 
Table 3

Health-Related Quality of Life

\begin{tabular}{lcc}
\hline Parameters & Base Estimate (Range) & References \\
\hline Well & 1 & Authors' assumption \\
\hline Steatosis & $1.0(0.86-1)$ & {$[41]$, Author's assumption } \\
\hline NASH & $0.85(0.84-0.86)$ & {$[41]$} \\
\hline Fibrosis & $0.84(0.83-0.85)$ & {$[41]$} \\
\hline Cirrhosis & $0.80(0.65-0.89)$ & {$[21 ; 43 ; 44 ; 46]$} \\
\hline Decompensated cirrhosis & $0.60(0.46-0.81)$ & {$[21 ; 43 ; 44 ; 46]$} \\
\hline Hepatoma & $0.73(0.50-0.80)$ & {$[43]$} \\
\hline Surgical resection (1st month) & $0.73(0.62-0.84)$ & {$[107]$} \\
\hline Liver transplant (1st year) & $0.69(0.62-0.86)$ & {$[44 ; 45 ; 108]$} \\
\hline Liver transplant (after transplant) & $0.80(0.79-0.83)$ & {$[108]$} \\
\hline
\end{tabular}

Abbreviations: NASH, nonalcoholic steatohepatitis. 


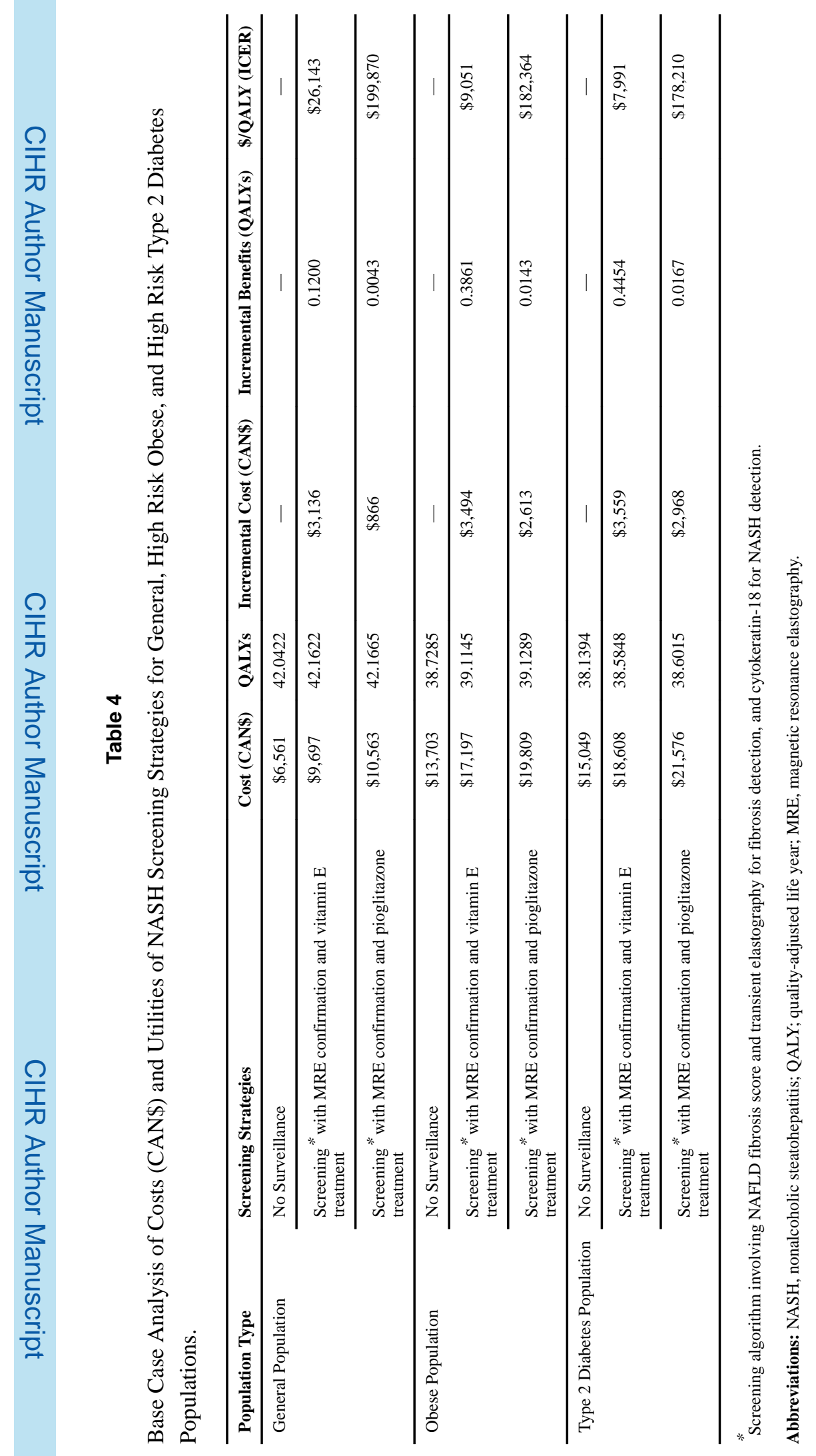

Eur Radiol. Author manuscript; available in PMC 2016 April 19. 